\title{
Relationship between plasma zinc and antibacterial innate immune function in the elderly
}

\author{
Sarah Forster ${ }^{1}$, Dan Flowers ${ }^{1}$, Gemma Foulds ${ }^{1}$, Laura Jones ${ }^{1}$, Tracey Young ${ }^{1}$, Kay Hopkinson ${ }^{1}$, \\ John Saxton ${ }^{2}$, Hilary Powers ${ }^{1}$, Graham Pockley ${ }^{1}$ and Elizabeth Williams ${ }^{1}$ \\ ${ }^{1}$ The University of Sheffield, Sheffield, UK and ${ }^{2}$ Sheffield Hallam University, Sheffield, UK
}

\begin{tabular}{|c|c|c|c|c|c|c|c|c|}
\hline \multicolumn{9}{|c|}{$\begin{array}{l}\text { Ageing is associated with impaired immunity and an increased sus } \\
\text { amongst older individuals in the } \mathrm{UK}^{(2)} \text {, and as nutritional status also } \\
\text { impact of such deficiencies on immune function in the elderly. } \\
\text { Participants ( } n 85 \text {, free-living, } 65-85 \text { years old) were recruited } \\
\text { including writing to potential participants from general practitioner } \\
\text { analysed using inductively-coupled plasma MS (HP } 4500 \text {; Agilent, Cl } \\
\text { using standard techniques, and phagocytic responses of monocytes to } \\
\text { synthetic peptide that mimics bacterially-derived peptides) and phorb } \\
\text { uated by whole-blood flow cytometry. }\end{array}$} \\
\hline \multirow{3}{*}{$\begin{array}{l}\text { Quartile... } \\
\text { Zn }(\mu \mathrm{mol} / \mathrm{l}) \ldots\end{array}$} & \multirow{2}{*}{\multicolumn{2}{|c|}{$\begin{array}{c}1(n 20) \\
<10.4\end{array}$}} & \multirow{2}{*}{\multicolumn{2}{|c|}{$\begin{array}{c}2(n 21) \\
10.4-11.7\end{array}$}} & \multirow{2}{*}{\multicolumn{2}{|c|}{$\begin{array}{c}3(n 19) \\
11.7-12.6\end{array}$}} & \multirow{2}{*}{\multicolumn{2}{|c|}{$\begin{array}{c}4(n 20) \\
>12.6\end{array}$}} \\
\hline & & & & & & & & \\
\hline & Median & IQR & Median & IQR & Median & $\mathrm{IQR}$ & Median & IQR \\
\hline \multicolumn{9}{|c|}{ Differential leucocyte count $\left(\times 10^{9} / 1\right)$} \\
\hline Neutrophils & 3.8 & $3.0-4.3$ & 4.0 & $2.9-4.7$ & 3.9 & $3.1-4.7$ & 4.0 & $3-5$ \\
\hline Lymphocytes & 1.7 & $1.6-2.1$ & 2.1 & $1.7-2.7$ & 2.1 & $1.7-2.5$ & 2.4 & $1.8-2.7$ \\
\hline Monocytes & 0.3 & $0.3-0.5$ & 0.4 & $0.4-0.5$ & 0.4 & $0.3-0.5$ & 0.4 & $0.3-0.5$ \\
\hline Eosinophils & 0.2 & $0.1-0.4$ & 0.2 & $0.1-0.3$ & 0.2 & $0.1-0.2$ & 0.2 & $0.1-0.3$ \\
\hline Basophils & 0.05 & $0.03-0.07$ & 0.04 & $0.03-0.06$ & 0.04 & $0.03-0.05$ & 0.05 & $0.03-0.06$ \\
\hline \multicolumn{9}{|c|}{ Monocyte phagocytosis, fluorescent intensity } \\
\hline Unstimulated & 31 & $22-39$ & 32 & $25-40$ & 31 & $24-63$ & 27 & $24-33$ \\
\hline+ E. coli & 262 & $152-364$ & 265 & $122-421$ & 223 & $126-388$ & 248 & $170-326$ \\
\hline \multicolumn{9}{|c|}{ Monocyte oxidative burst, fluorescent intensity } \\
\hline Unstimulated & 14 & $13-16$ & 15 & $14-17$ & 15 & $13-16$ & 16 & $14-18$ \\
\hline+ fMLP & 16 & $14-17$ & 15 & $14-17$ & 16 & $15-17$ & 16 & $13-18$ \\
\hline + PMA & 22 & $17-37$ & 27 & $17-37$ & 19 & $13-25$ & 19 & $15-28$ \\
\hline+ E. coli & 22 & $17-25$ & 25 & $21-35$ & 17 & $15-27$ & $17 *$ & $14-25$ \\
\hline
\end{tabular}

IQR, interquartile range. Median value was significantly different from that for quartile 1 (Kruskal-Wallis): $* P=0.012$.

The median plasma $\mathrm{Zn}$ concentration in this cohort of elderly individuals was 11.7 (interquartile range 10.4-12.6) $\mu \mathrm{mol} / \mathrm{l}$ and this value compares with $14.3 \mu \mathrm{mol} / \mathrm{l}$, which has been reported in the National Diet and Nutrition Survey for older people ${ }^{(2)}$. Although lymphocyte numbers were higher in individuals in the upper quartile for plasma $\mathrm{Zn}$ concentrations, this difference was not significant. No other relationship between $\mathrm{Zn}$ concentration and leucocyte counts were apparent. Furthermore, there was no relationship between plasma $\mathrm{Zn}$ and any of the monocyte functional variables, except for a reduced ability to generate an oxidative-burst response to $E$. coli in individuals having the highest plasma $\mathrm{Zn}$ concentrations (Table). Dietary data has been collected and will be analysed to give information on dietary intakes of zinc.

Overall, the present study fails to identify a robust relationship between plasma $\mathrm{Zn}$ status and measures of innate immune function in the elderly; this finding may reflect the relative homogeneity of plasma $\mathrm{Zn}$ in this cohort.

This study (N05068) was funded by the Food Standards Agency.

1. Miller RA (1996) Science 273, 70-74.

2. Finch S, Doyle W, Lowe C, Bates C, Prentice A, Smithers G \& Clarke C (1998) National Diet and Nutrition Survey. London: The Stationery Office.

3. Lesourd BM (1997) Am J Clin Nutr 66, 478S-84S. 